\title{
Electroweak and QCD Combined Fit of HERA-II Data
}

\author{
Gang LI \\ On behalf of the H1 collaboration, \\ Laboratoire de l'Accélérateur Linéaire, Université Paris-Sud 11, \\ IN2P3/CNRS, BP34, 91898 Orsay, France
}

\begin{abstract}
We report a new Electroweak and QCD combined fit based on H1 measurements. Due to the polarizations of electron and positron beams at HERA-II, the precision of the Electroweak couplings of the light quarks to $Z^{0}$ has been improved significantly.
\end{abstract}

\section{Introduction}

The deep inelastic scattering (DIS) of leptons off nucleons has played an important role in revealing the structure of matter, in the discovery of weak neutral current interactions and in the foundation of the Standard Model (SM) as the theory of strong and Electroweak (EW) interactions. At HERA, the first lepton-proton collider ever built, the study of DIS has been pursued since 1992 over a wide kinematic range. In terms of $Q^{2}$, the negative four-momentum transfer squared, the kinematic coverage includes the region where the electromagnetic and weak interactions become of comparable strength. Both charged current (CC) and neutral current (NC) interactions occur in ep collisions and are studied by the two collider experiments H1 and ZEUS. Many QCD analyses of HERA data have been performed to determine the strong interaction coupling constant $\alpha_{s}[2,3,4]$ and parton distribution functions (PDFs) $[3,5,6]$. In EW analyses, the $\mathrm{W}$ boson mass value has been determined from the charged current data at high $Q^{2}[5,7,8,9,10,11,12]$, and from combined fit in [13].

Based solely on the precise data recently published by $\mathrm{H} 1[2,5,6,9]$ and new data of HERA-II, a combined QCD and EW analysis is performed here and parameters of the Electroweak theory are determined. The published data have been taken by the $\mathrm{H} 1$ experiment in the first phase of operation of HERA (HERA-I) with unpolarised $e^{+}$and $e^{-}$beams and correspond to an integrated luminosity of $100.8 p b^{-1}$ for $e^{+} p$ and $16.4 p b^{-1}$ for $e^{-} p$, respectively. The new data were taken in the second phase of operation (HERA-II, including 2003-2005) with polarized $e^{+}$and $e^{-}$beams and correspond to an integrated luminosity of $47.6 p b^{-1}$ for $e^{+} p$ and $98.2 p b^{-1}$ for $e^{-} p$ respectively.

At HERA, the NC interactions at high $Q^{2}$ receive contributions from $\gamma Z$ interference and $Z^{0}$ exchange. Thus the NC data can be used to extract the weak couplings of up- and down-type quarks to the $Z^{0}$ boson. At high $Q^{2}$ and high $x$, where the $\mathrm{NC} e^{ \pm} p$ cross sections are sensitive to these couplings, the up- and down-type quark distributions are dominated by the light $\mathrm{u}$ and $\mathrm{d}$ quarks. Therefore, this measurement can be considered to determine the light quark couplings. The $\mathrm{CC}$ cross section data help to disentangle the up and down quark distributions. At HERA-II, the $e^{ \pm}$beams are polarized. For neutral current, the cross section can be expressed as:

$$
\begin{aligned}
\frac{d^{2} \sigma_{N C}^{ \pm}}{d x d Q^{2}} & =\frac{2 \pi \alpha^{2}}{x Q^{4}} \phi_{N C}^{ \pm}, \\
\phi_{N C}^{ \pm} & =Y_{+} \tilde{F}_{2} \mp Y_{-} x \tilde{F}_{3}-y^{2} \tilde{F}_{L} .
\end{aligned}
$$


Here we neglect the contribution of $\tilde{F}_{L}$, the other two structure functions are:

$$
\begin{aligned}
\tilde{F}_{2}^{ \pm} & \simeq F_{2}^{\gamma}-\left( \pm P_{e} a_{e}\right) \chi_{Z} F_{2}^{\gamma Z}, \\
x \tilde{F}_{3}^{ \pm} & \simeq-\left(a_{e}\right) \chi_{Z} x F_{3}^{\gamma Z},
\end{aligned}
$$

where the $\chi_{Z}$ is the propagator of the $Z$ boson. Since $\chi_{Z} \gg \chi_{Z}^{2}$ and $v_{e} \simeq 0.05$, the pure $Z^{0}$ terms and the corresponding terms of $v_{e}$ have been neglected here.

In the quark parton model, the $\gamma Z$ interference terms can be expressed as :

$$
\begin{aligned}
F_{2}^{\gamma Z} & =\sum 2 e_{i} v_{i}\left(x q_{i}+x \bar{q}_{i}\right), \\
x F_{3}^{\gamma Z} & =\sum 2 e_{i} a_{i}\left(x q_{i}-x \bar{q}_{i}\right) .
\end{aligned}
$$

According to the formulae 1 and 2, we can see that the polarizations of $e^{ \pm}$put more constraints to the Electroweak couplings. So we can expect high sensitivity of the couplings from the polarized data of HERA-II.

\section{Data sets and fit strategies}

The QCDFit [14] package has been used in this work. The combined EW-QCD analysis follows the same fit procedure as used in $[6,13]$. The QCD analysis is performed using the DGLAP evolution equations [15] at NLO [16] in the MS renormalisation scheme. The contributions of the heavy quarks are taken into account, including the top quark. All quarks are taken to be massless when $Q^{2}$ is above their mass thresholds.

Fits are performed to the measured cross sections by fixing the strong coupling constant to be equal to $\alpha_{s}\left(M_{Z}^{2}\right)=0.1185$. The analysis uses an $x$-space program developed within the H1 Collaboration [17]. In the fit procedure, a $\chi^{2}$ function which is defined in [2] is minimized. The minimization takes into account correlations between data points caused by systematic uncertainties [6].

In the fits, five PDFs - gluon, $x U, x D, x \bar{U}$ and $x \bar{D}$ are defined by 10 free parameters as in $[6,13]$. For all fits, the PDFs obtained here are consistent with those from the H1 PDF 2000 fit [6].

The analysis performed here uses not only HERAI data sets as in [6], but also the data sets obtained by HERA-II(including 2003-2005 data) which has polarized electron and positron beams. These data cover a Bjorken $x$ range from $3 \times 10^{-5}$ to 0.65 depending on $Q^{2}$. The fit using a toy Monte Carlo data shows that the polarization will improve the Electroweak couplings significantly, especially for the up quark (See figure 1).

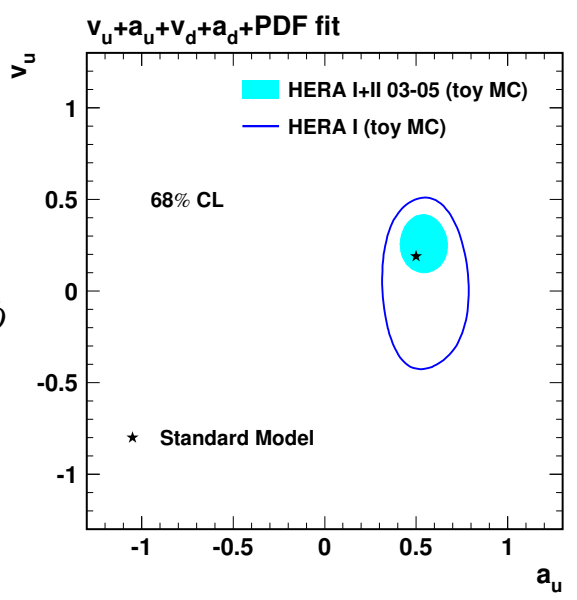

Figure 1: Comparison of fits using a toy Monte Carlo, 94-00 data and 9405 data. 


\section{Fit results}

In this analysis "fit $v_{u}-a_{u}-v_{d}-a_{d}-\mathrm{PDF}$ ", the vector and axial-vector dressed couplings of $u$ and $d$ quarks together with PDF are treated as free parameters. The effect of the $u$ and $d$ correlation is studied by fixing either $u$ or $d$ quark couplings to their SM values fits " $v_{u}-a_{u}-\mathrm{PDF}$ " and " $v_{d}-a_{d}-\mathrm{PDF} "$.

The results from this analysis are compared in figure 2 with the previous results of HERA-I [13]. We can see that the precision of $u$ quark couplings are improved significantly as expected, which is due to the contribution of polarized $e^{ \pm}$beams.
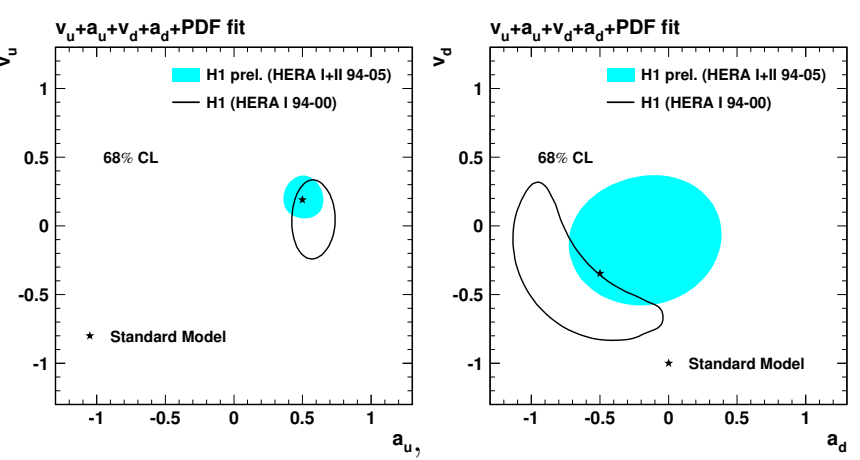

Figure 2: Fit results: contours of vector- and axialcouplings of $u$ and $d$ quark, compared with HERA-I.

The precision is better for the $u$ quark as expected. The superior precision for $a_{u}$ comes from the $\gamma Z$ interference contribution $x F_{3}$. And the improvement of $v_{u}$ comes from the $\gamma Z$ interference term $F_{2}^{\gamma Z}$ due to non zero polarization of $e^{ \pm}$. Similarly the d-quark couplings $v_{d}$ and $a_{d}$ are also constrained by these terms but the smaller electrical charge decreases the effect of these constraints.

The results from this analysis are also compared in figure 3 with similar results obtained previously by the CDF experiment [18]. The HERA determination of the $u$ quark couplings has better precision than that from the Tevatron. These determinations are sensitive to $u$ and $d$ quarks separately, contrary to other measurements of the light quark- $Z^{0}$ couplings in $\nu N$ scattering [19] and atomic parity violation [20] on heavy nuclei. They also resolve any sign ambiguity and the ambiguities between $v_{u}$ and $a_{u}$ of the determinations based on observables measured at the $Z^{0}$ resonance [21] at LEP.

\section{Summary}

The vector and axial-vector weak neutral current couplings of $u$ and $d$ quarks to the $Z^{0}$ boson have been determined at HERA. The precision of $u$ quark couplings has been improved significantly with respect to the HERA-I results. Also better precision of the $u$ quark couplings was achieved compared with the CDF results [18], and it has no sign ambiguities such as those of LEP [21]. All results are consistent with the Elec-
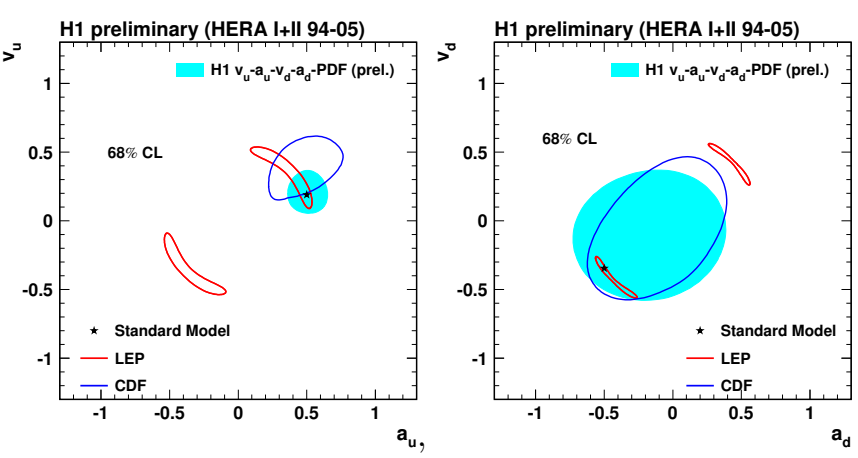

Figure 3: Fit results: contours of vector- and axialcouplings of $u$ and $d$ quark, compared with those of LEP and CDF. 
troweak Standard Model.

Further improvement in presision is expected as more HERA-II data will be analysed and included in the combined fit.

\section{References}

[1] Slides: http://indico. cern. ch/contributionDisplay . py? contribId=38\&sessionId=14\&conf Id=9499

[2] C. Adloff et al. [H1 Collaboration], Eur. Phys. J. C21 33 (2001).

[3] S. Chekanov et al. [ZEUS Collaboration], Phys. Rev. D67 012007 (2003).

[4] S. Chekanov et al. [ZEUS Collaboration], Eur. Phys. J. C42 1 (2005).

[5] C. Adloff et al. [H1 Collaboration], Eur. Phys. J. C13 609 (2000).

[6] C. Adloff et al. [H1 Collaboration], Eur. Phys. J. C30 1 (2003).

[7] T. Ahmed et al. [H1 Collaboration], Phys. Lett. B324 241 (1994).

[8] S. Aid et al. [H1 Collaboration], Phys. Lett. B379 (1996) 319

[9] C. Adloff et al. [H1 Collaboration], Eur. Phys. J. C19 (2001) 269

[10] J. Breitweg et al. [ZEUS Collaboration], Eur. Phys. J. C12 411 (2000); [Erratum ibid. B27 305 (2003)].

[11] S. Chekanov et al. [ZEUS Collaboration], Phys. Lett. B539 197 (2002); [Erratum ibid. B552 (2003) 308].

[12] S. Chekanov et al. [ZEUS Collaboration], Eur. Phys. J. C32 1 (2003).

[13] A. Aktas et al. [H1 Collaboration], Phys. Lett. B632 35 (2006).

[14] Manual: http://h1.web.lal.in2p3.fr/divers/psfiles/notice.ps

[15] Y.L. Dokshitzer, Zh. Eksp. Teor. Fiz. 73 (1977) 1216; V.N. Gribov and L.N. Lipatov, Yad. Fiz. 15 1218 (1972) ; V.N. Gribov and L.N. Lipatov, Yad. Fiz. 15781 (1972); G. Altarelli and G. Parisi, Nucl. Phys. B126 298 (1977) .

[16] W. Furmanski and R. Petronzio, Phys. Lett. B97 437 (1980).

[17] C. Pascaud and F. Zomer, LAL preprint, LAL 95-05 (1995); C. Pascaud and F. Zomer, "A fast and precise method to solve the Altarelli-Parisi equations in x space" [hep-ph/0104013].

[18] D. Acosta et al. [CDF Collaboration], Phys. Rev. D71 052002 (2005)

[19] G.P. Zeller et al. [NuTeV Collaboration], Phys. Rev. Lett. 88091802 (2002) [Erratum-ibid. 90239902 (2003)].

[20] S.C. Bennett and C.E. Wieman, Phys. Rev. Lett.82 2484 (1999).

[21] LEP and SLD Electroweak working groups, "Combination of Preliminary Electroweak Measurements and Constraints on the Standard Model"; http://lepewwg.web.cern.ch/LEPEWWG/plots/summer2004/. 